\title{
THE BUCKLING OF A FREE EDGE OF AN AXIALLY-COMPRESSED CIRCULAR CYLINDRICAL SHELL*
}

\author{
$\mathrm{BY}$ \\ W. NACHBAR AND N. J. HOFF \\ Stanford University, Stanford, California
}

1. Introduction. It is well known that the classical theory of the stability of thinwalled circular cylindrical shells subjected to uniform axial compression yields critical stresses which amount to two to four times the buckling stresses observed in experiment. This is the reason why non-linear theories [1, 2] t have been developed during the last twenty-odd years. It is also common knowledge, at least among experimentalists, that buckles always form along the loaded circular edges of a thin-walled cylindrical specimen before any deformations can be observed in the remainder of the specimen unless the edges are reinforced by stiffeners or are cast in a metal plate. The early buckling of the edges is usually attributed [3] to inaccuracies of the test set-up in regard to edge support and load application. Finally the buckles of the classical theory cover uniformly the entire surface of the cylinder while the experimental buckles are always rapidly attenuated.

In the present paper the classical linear equations are solved for free-edge boundary conditions. The results are in much better agreement with experiment than the classical solution. In particular, the critical stress is 37 percent of the accepted value, the circumferential wave length is in reasonable agreement with observation, and in the axial direction the displacements are attenuated rapidly. The authors do not maintain that the axial loading of free edges as stipulated in the present paper is a true representation of the conditions prevailing in the usual laboratory test; but neither is the customary simple support condition a true representation. At any rate, the present study explains why the loaded circular edges of the shell buckle before the rest if they are not reinforced.

A similar buckling theory for axisymmetric deformations was presented by one of the authors in his von Kármán 80th Anniversary Lecture [2]. The possibility of edge buckling under a low critical stress occurred to him when he studied the dependence of the influence coefficients of thin-walled spherical shells on the internal pressure as derived by the other author [4]. The axially-symmetric buckling of the axially-loaded free edges of thin-walled circular cylindrical shells was found to take place at one-half the conventional value of the critical stress.

2. Formulation of the problem. The classical equations governing the displacements of the midsurface of the shell from a state of uniform compression will be used in a non-dimensional form. The present version of the equations is based on the simplifications suggested by Donnell [3]. A non-dimensional small parameter $\epsilon^{*}$ and a characteristic length $a \epsilon^{* 1 / 2}$ are introduced. Their definitions are

$$
\begin{aligned}
\epsilon^{*} & \equiv h / 2 a\left[3\left(1-\nu^{2}\right)\right]^{1 / 2}, \\
a \epsilon^{* 1 / 2} & \equiv\left[12\left(1-\nu^{2}\right)\right]^{-1 / 4}(h a)^{1 / 2},
\end{aligned}
$$

*Received January 8, 1962. This work was performed at Stanford University with the sponsorship of the National Aeronautics and Space Administration under grant NsG.93-60.

$\dagger$ Numbers in square brackets refer to the references at the end of the paper. 


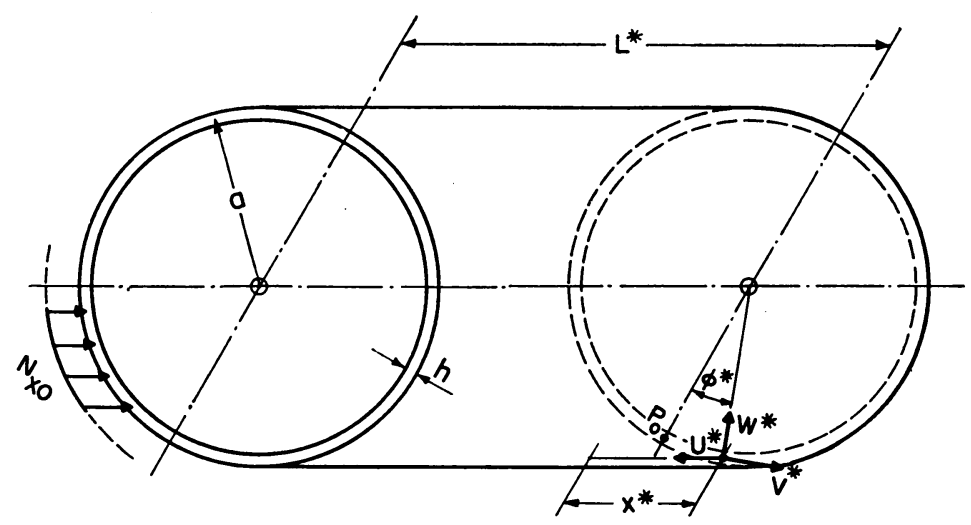

Fig. 1. Cylindrical shell: dimensions, coordinates, displacements and loads.

where $a$ is the radius of the midsurface of the cylinder, $h$ the wall thickness and $\nu$ is Poisson's ratio. With $x^{*}$ and $\varphi^{*}$ denoting the axial and circumferential coordinates (see Fig. 1), and $u^{*}, v^{*}, w^{*}$ the displacements of a point on the midsurface from the state of uniform compression, the non-dimensional quantities used in the equations are defined as

$$
\begin{aligned}
x \equiv x^{*} / a \epsilon^{* 1 / 2}, & \varphi \equiv \varphi^{*} / \epsilon^{*^{1 / 2}}, \\
u \equiv u^{*} / a \epsilon^{*^{1 / 2}}, & v \equiv v^{*} / a \epsilon^{*^{1 / 2}}, \quad w \equiv w^{*} / a .
\end{aligned}
$$

The initial uniform axial stress is $\sigma_{x 0}$. It appears in the equations implicitly in the form of a stress ratio $\rho$ defined as

$$
\rho \equiv \sigma_{x 0} / \sigma_{c 1} \equiv \sigma_{x 0} / 2 \epsilon^{*} E,
$$

which is the ratio of the uniform applied axial compressive stress to the critical stress $\sigma_{c 1}$ of the classical theory for simply supported edges ( $E$ is Young's modulus.) Use is also made of a non-dimensional stress function $f$ related to the conventional stress function $F^{*}$ for the stress resultants [1] by the equation

$$
f=\epsilon^{*} \rho \varphi^{2}+F^{*} / a^{2} \epsilon^{*} E h .
$$

The equations governing the non-dimensional displacement $w$ are

$$
\begin{aligned}
\nabla^{4} w & =-2 \rho w_{. x x}+f_{, x x}, \\
\nabla^{4} f & =-w_{. x x}, \\
\nabla^{2}() & \equiv()_{, x x}+()_{, \varphi \varphi} .
\end{aligned}
$$

The non-dimensional axial and circumferential displacements $u$ and $v$ are determined by the equations

$$
\begin{aligned}
& \nabla^{4} u=\nu w_{, x x x}-w_{, x \varphi \varphi}, \\
& \nabla^{4} v=(2+\nu) w_{, x x \varphi}+w_{, \varphi \varphi \varphi} .
\end{aligned}
$$

For the present investigation, expressions are needed for the stress resultants and stress couples acting upon the circular edge of the cylindrical shell cut by a plane normal 
to the $x^{*}$ direction. The relevant quantities are the axial normal stress resultant $N_{x}^{*}$, taken as positive in tension, the shear stress resultant $N_{x \varphi}^{*}$ directed along the edge of the midsurface, the transverse shear stress resultant $Q_{x}^{*}$, the bending stress couple $M_{x}^{*}$ and the twisting stress couple $M_{x \varphi}^{*}$. The well-known [5] expressions for these stress resultants and stress couples become with the present notation

$$
\begin{gathered}
N_{x}^{*}=E h\left[f_{, \varphi \varphi}-2 \epsilon^{*} \rho\right], \quad N_{x \varphi}^{*}=-E h f_{, x \varphi} \\
M_{x}^{*}=-E h a \epsilon^{*}\left(w_{, x x}+\nu w_{, \varphi \varphi}\right) \\
M_{x \varphi}^{*}=E h a \epsilon^{*}(1-\nu) w_{, x \varphi} \\
Q_{x}^{*}=-E h \epsilon^{*^{1 / 2}}\left(w_{, x x x}+w_{, x \varphi \varphi}\right)
\end{gathered}
$$

The expressions for $T_{x}^{*}$ and $S_{x}^{*}$, the total edge resultants, become with the present notation

$$
\begin{aligned}
& T_{x}^{*}=Q_{x}^{*}-\frac{\partial M_{x \varphi}^{*}}{a \partial \varphi^{*}}=-E h \epsilon^{*^{1 / 2}}\left[w_{, x x x}+(2-\nu) w_{, x \varphi}\right] \\
& S_{x}^{*}=N_{x \varphi}^{*}+M_{x \varphi}^{*} / a=-E h\left[f_{, x \varphi}-\epsilon^{*}(1-\nu) w_{, x \varphi}\right]
\end{aligned}
$$

The equilibrium conditions at the deformed edge of the prestressed shell will now be derived. If it is assumed that the applied initial axial force distribution $N_{x 0}^{*}$ at an edge point (Fig. 1) is maintained fixed in magnitude and direction while the edge point undergoes a small additional displacement, then $N_{x 0}^{*}$ will contribute also to the shear reactions. Consider the generic point $P_{0}$, located on the midsurface at the edge of the shell after application of the prestress (Fig. 1). Subsequently, $P_{0}$ is deformed to the position $P_{1}$, as shown in Fig. 2a. A right-handed coordinate system at $P_{0}$ is determined by the axial coordinate $x^{*}$, the coordinate $z^{*}$ taken along the inwards-directed normal, and the coordinate $y^{*}$ taken along the tangent to the midsurface. Let the right-handed triad of unit vectors at $P_{1},\left\{\mathbf{i}_{x}, \mathbf{i}_{y}, \mathbf{i}_{z}\right\}$, be parallel respectively to the $x^{*}, y^{*}, z^{*}$ directions at $P_{0}$. Let the unit vector $\mathbf{j}_{x}$ be tangent to the deformed generator of the midsurface at $P_{1}$, and let the unit vector $\mathbf{j}_{z}$ lie along the inwards-directed normal to the deformed midsurface at $P_{1}$. Then $\mathbf{j}_{v}$ is taken such that $\left\{\mathbf{j}_{x}, \mathbf{j}_{v}, \mathbf{j}_{z}\right\}$ is a right-handed triad of unit vectors at $P_{1}$.

Love [6] has derived the linear transformation linking these two vector triads. If we write

$$
\begin{aligned}
& \mathbf{j}_{x}=l_{1} \mathbf{i}_{x}+m_{1} \mathbf{i}_{y}+n_{1} \mathbf{i}_{z}, \\
& \mathbf{j}_{y}=l_{2} \mathbf{i}_{x}+m_{2} \mathbf{i}_{y}+n_{2} \mathbf{i}_{z}, \\
& \mathbf{j}_{z}=l_{3} \mathbf{i}_{x}+m_{3} \dot{\mathbf{i}}_{y}+n_{3} \mathbf{i}_{z},
\end{aligned}
$$

then the components of the orthogonal transformation matrix for the cylindrical shell become, in the present notation

$$
\begin{gathered}
l_{1}=1, \quad m_{1}=v_{, x}, \quad n_{1}=w_{, x} / \epsilon^{*^{1 / 2}}, \quad l_{2}=-m_{1} \\
m_{2}=1, \quad n_{2}=\epsilon^{*^{1 / 2}} v+\left(w_{, \varphi} / \epsilon^{*^{1 / 2}}\right), \quad l_{3}=-n_{1} \\
m_{3}=-n_{2}, \quad n_{3}=1 .
\end{gathered}
$$




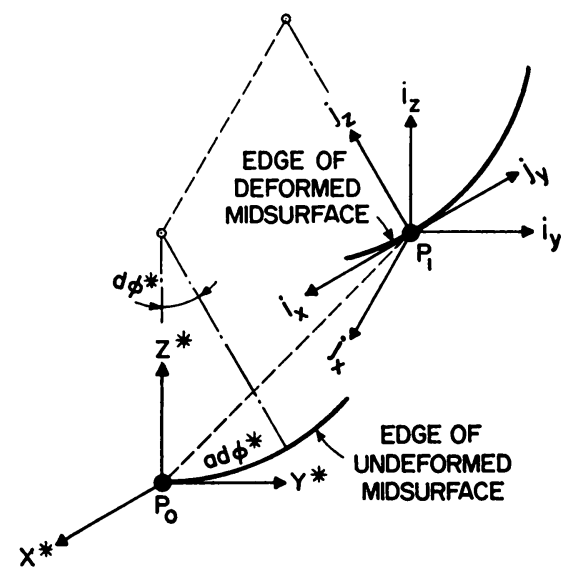

(a)

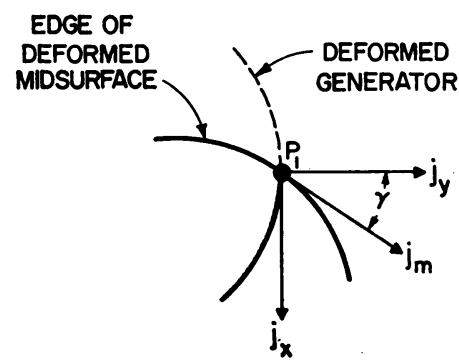

(b)

FIG. 2. (a) and (b)-Unit vector relations at an edge of a deformed cylindrical shell.

The vector deformation from $P_{0}$ to $P_{1}$ is, by definition:

$$
u^{*} \mathbf{i}_{x}+v^{*} \mathbf{i}_{\nu}+w^{*} \mathbf{i}_{z} .
$$

The unit vector $\mathbf{j}_{m}$ is defined to be tangent to the edge of the deformed midsurface at $P_{1}$ (Fig. 2b). Then $\mathbf{j}_{m}$ lies in the plane determined by $\mathbf{j}_{x}$ and $\mathbf{j}_{y}$; it has been sheared through an angle $\gamma$ (engineering shear strain) out of the plane determined by $\mathbf{j}_{\nu}$ and $\mathbf{j}_{\mathbf{z}}$. We have

$$
\gamma=v_{, x}+u_{, \varphi}
$$

and so $\mathbf{j}_{m}$ is expressed as

$$
\mathbf{j}_{m}=\gamma \mathbf{j}_{x}+\mathbf{j}_{y} .
$$

With the usual sign convention [5] the initial compressive force distribution is represented by the vector $\mathbf{F}_{0}$ :

$$
\mathbf{F}_{0}=-N_{x 0}^{*} \mathbf{i}_{x} .
$$

When the deformations $w^{*}, v^{*}, u^{*}$ take place, an additional force distribution $\mathbf{F}_{1}$ may be applied, in general, to the edge: 


$$
\mathbf{F}_{1}=F_{1 x}^{*} \mathbf{i}_{x}+F_{1 y}^{*} \mathbf{i}_{y}+F_{1 z}^{*} \mathbf{i}_{z} .
$$

The edge reactions must be in equilibrium with the total of the applied force distributions. If the edge reactions are represented by the vector $F$ as

$$
\mathbf{F}=N_{x}^{*} \mathbf{j}_{x}+T_{x}^{*} \mathbf{j}_{z}+S_{x}^{*} \mathbf{j}_{m},
$$

then three scalar equations of equilibrium at the edge are given by the vector equation

$$
\mathbf{F}=\mathbf{F}_{0}+\mathbf{F}_{1} \text {. }
$$

The free-edge condition is the special case of Eq. (19) in which $F_{1}$ is zero. The following relation can be derived from Eqs. (12) and (13):

$$
\mathbf{i}_{x}=l_{1} \mathbf{j}_{x}+l_{2} \mathbf{j}_{y}+l_{3} \mathbf{j}_{z}=\mathbf{j}_{x}-v_{, x} \mathbf{j}_{y}-\epsilon^{*-1 / 2} w_{, x} \mathbf{j}_{z} .
$$

Because of Eqs. (15), (18) and (20), Eq. (19) may be written for the free-edge condition as

$$
\begin{gathered}
\left(N_{x}^{*}+N_{x 0}^{*}+\gamma S_{x}^{*}\right) \mathbf{j}_{x}+\left(S_{x}^{*}-N_{x 0}^{*} v_{. x}\right) \mathbf{j}_{y}, \\
+\left[T_{x}^{*}-N_{x 0}^{*} \epsilon^{*^{-1 / 2}} w_{. x}\right] \mathbf{j}_{z}=0 .
\end{gathered}
$$

When substitution from Eqs. $(10 a, b)$ and $(11 a, b)$ is made, three scalar equilibrium equations are derived from Eq. (21):

$$
\begin{gathered}
f_{. \varphi \varphi}=0, \\
f_{. x \varphi}-\epsilon^{*}(1-\nu) w_{. x \varphi}+2 \epsilon^{*} \rho v_{. x}=0, \\
w_{. x x x}+(2-\nu) w_{. x \varphi \varphi}+2 \rho w_{. x}=0 .
\end{gathered}
$$

In deriving Eqs (22), use was made of the identity $N_{x 0}^{*} / E h \equiv-2 \epsilon^{*} \rho$, and it was assumed that $\epsilon^{*}>0$. Moreover, the term $\gamma\left[f_{, x \varphi}-\epsilon^{*}(1-\nu) w_{, x \varphi}\right]$ was neglected in Eq. (22a); this is consistent with the linearization of the Donnell equations.

The free-edge condition also prescribes $M_{x}^{*}=0$, and it follows from Eq. (10c) that

$$
w_{, x x}+\nu w_{, \varphi \varphi}=0 .
$$

3. Solution of the Buckling Problem. The boundary-value problem considered is that of a thin-walled shell $\left(\epsilon^{*} \ll 1\right)$ of finite length $L^{*}$; the non-dimensional length $L$ is defined as

$$
L \equiv L^{*} / \pi a \epsilon^{* 1 / 2}
$$

Equations (5) and (6) are to hold for $0<x<\pi L$, and free-edge buckling Equations $(22 \mathrm{a}, \mathrm{b}, \mathrm{c})$ and (23) are to hold at $x=0$ and at $x=\pi L$. These equations hold for all $\varphi$, and solutions are required to be periodic in $\varphi$ with period $2 \pi / \epsilon^{*^{1 / 2}}$. This problem is homogenous. Non-trivial solutions are sought where $f$ has the form ("take the real part" is understood)

$$
f(x, \varphi)=C \exp \left[p x+\frac{1}{2} i \Lambda^{1 / 2} \varphi\right] .
$$

$C$ and $p$ are complex numbers, $i$ is the imaginary unit, $n$ is an integer which is the number of complete waves in the circumferential direction, and $\Lambda \equiv 4 \epsilon^{*} n^{2}$. It then follows from Eqs. (6) and (25) that $w$ must be of the form

$$
w(x, \varphi)=-\left(p^{2}-\Lambda / 4\right)^{2} p^{-2} f(x, \varphi)
$$


Equations (25) and (26) satisfy Eq. (5) only if $p$ is a root of

$$
\left(p^{2}-\Lambda / 4\right)^{4}+2 \rho p^{2}\left(p^{2}-\Lambda / 4\right)^{2}+p^{4}=0 .
$$

It was shown in an earlier paper [7] that, for $\Lambda>0$ and $\rho^{2}<1$, Eq. (27) has four distinct roots with negative real part. These roots are called $p_{K}, K=1,2,3,4$. If the bar is used to denote the complex conjugate, the four roots are:

$$
\begin{gathered}
p_{1} \equiv-\alpha_{1}+i \beta_{1}, \quad p_{2} \equiv-\alpha_{2}+i \beta_{2}, \quad p_{3}=\bar{p}_{1}, \quad p_{4}=\bar{p}_{2} \\
\alpha_{1}=\frac{1}{2^{3 / 2}}\left[(c-\rho+\Lambda)^{1 / 2}+(1-\rho)^{1 / 2}\right] \\
\beta_{1}=-\frac{1}{2^{3 / 2}}\left[(1+\rho)^{1 / 2}+(c+\rho-\Lambda)^{1 / 2}\right] \\
\alpha_{2}=\frac{1}{2^{3 / 2}}\left[(c-\rho+\Lambda)^{1 / 2}-(1-\rho)^{1 / 2}\right] \\
\beta_{2}=\frac{1}{2^{3 / 2}}\left[(1+\rho)^{1 / 2}-(c+\rho-\Lambda)^{1 / 2}\right] \\
c \equiv+\left[1-2 \rho \Lambda+\Lambda^{2}\right]^{1 / 2}
\end{gathered}
$$

There are also four distinct roots of Eq. (27) with positive real part.

It will be assumed that $L$ is large enough so that the amplitude of $w$ in the edgebuckling solution of the boundary-value problem decays to a relatively small value within a non-dimensional distance from an edge small in comparison to $\pi L / 2$. The validity of this assumption for shells of practical dimensions is apparent from the edge-buckling solution obtained below (see Fig. 4); this is further discussed in [8]. With this assumption, only that portion of the solution which has the edge $x=0$ as its boundary, and which decays for positive $x$, need be considered. The problem is then mathematically equivalent to the problem of the semi-infinite shell, and $f$ and $w$ can be represented as the following linear combinations of the exponential solutions in Eqs. (25) and (26), the $C_{K}, K=$ $1,2,3,4$, being arbitrary complex constants:

$$
\begin{aligned}
f(x, \varphi) & =\left[\sum_{K=1}^{4} C_{K} e^{p_{K^{x}}}\right] e^{(i / 2) \Lambda^{1 / 2} \varphi}, \\
w(x, \varphi) & =-\left[\sum_{K=1}^{4} C_{K}\left(p_{K}^{2}-\Lambda / 4\right)^{2} p_{K}^{-2} e^{p_{K^{x}}}\right] e^{(i / 2) \Lambda^{1 / 2} \varphi}
\end{aligned}
$$

A representation must also be found for $v(x, \varphi)$ in order to evaluate Eq. (22b). This follows from Eqs. (9) and (30):

$$
v(x, \varphi)=-\frac{1}{2} i \Lambda^{1 / 2}\left\{\sum_{K=1}^{4} C_{K}\left[(2+\nu) p_{K}^{2}-\Lambda / 4\right] p_{K}^{-2} e^{p_{K^{x}}}\right\} e^{(i / 2) \Lambda^{1 / 2} \varphi} .
$$

If the expressions from Eqs. (29), (30) and (31) are used in Eqs. (22) and (23) at $x=0$, the four linear algebraic equations that result are:

$$
\frac{1}{4} \Lambda \sum_{K=1}^{4} C_{K}=0
$$




$$
\begin{gathered}
-\frac{i}{2} \Lambda^{1 / 2} \sum_{K=1}^{4} C_{K} p_{K}\left\{1+\epsilon^{*}(1-\nu)\left(p_{K}^{2}-\Lambda / 4\right)^{2} p_{K}^{-2}-2 \epsilon^{*} \rho\left[2+\nu-\Lambda /\left(4 p_{K}^{2}\right)\right]\right\}=0 \\
\sum_{K=1}^{4} C_{K} p_{K}\left\{\left[p_{K}^{2}-\frac{1}{4}(2-\nu) \Lambda+2 \rho\right]\left(p_{K}^{2}-\Lambda / 4\right)^{2} p_{K}^{-2}\right\}=0 \\
\sum_{K=1}^{4} C_{K}\left(p_{K}^{2}-\frac{1}{4} \nu \Lambda\right)\left(p_{K}^{2}-\Lambda / 4\right)^{2} p_{K}^{-2}=0
\end{gathered}
$$

Equations (32) are further simplified through use of the following identities [7]:

$$
\left(p_{K}^{2}-\Lambda / 4\right)^{2} p_{K}^{-2}=-\rho+i \sqrt{1-\rho^{2}}, \quad K=1 \text { and } 2 .
$$

The absolute value of the right-hand side of Eq. (33) is unity for all $\rho$ in the interval $\rho^{2}<1$. Since $\epsilon^{*} \ll 1$, then the terms in curly brackets in the sum of Eq. (32b) can be approximated as

$$
1+\frac{\epsilon^{*} \rho \Lambda}{2 p_{K}^{2}}, \quad K=1,2,3,4
$$

The second term above is negligible in comparison with unity unless either $\epsilon^{*} \Lambda$ becomes of order unity or $p_{K}^{2}$ becomes small. The first situation implies $\Lambda \gg 1\left(n\right.$ of order $\left.\epsilon^{*^{-1}}\right)$. The edge-buckling solution obtained below and the experimental evidence [8] both show that $\Lambda$ is not in this range of values at buckling. Therefore, it can be assumed that $\epsilon^{*} \Lambda$ is of order $\epsilon^{*}$. The second situation is considered further, since $\left|p_{2}^{2}\right|$ and $\left|p_{4}^{2}\right|$ tend towards zero as $\Lambda$ tends towards zero for all $\rho^{2}<1$. This behavior can be deduced from Fqs. (28), or, more directly, from Fig. 1 of [7], from which it can also be observed that $\left|p_{1}^{2}\right|$ and $\left|p_{3}^{2}\right|$ remain bounded away from zero as $\Lambda$ tends towards zero.

An expansion of $p_{2}$ about $\Lambda=0$ has as its first term

$$
p_{2}=\frac{\Lambda}{4 \sqrt{2}}\left[-(1-\rho)^{1 / 2}+i(1+\rho)^{1 / 2}\right], \quad \Lambda \ll 1,
$$

so that for small $\Lambda$

$$
\frac{\epsilon^{*} \rho \Lambda}{2\left|p_{2}^{2}\right|} \doteq \frac{8 \epsilon^{*} \rho}{\Lambda}=\frac{2 \rho}{n^{2}}
$$

Since $\rho$ is at most unity, then, if $n$ is greater than 4 , the term on the right of Eq. (35) is negligible compared to unity. It is expected that the solution will be such that $n$ satisfies this condition; an equivalent condition is also a requirement for the validity of the approximations involved in the derivation of Eqs. (5), (6), (8) and (9).

Therefore, for sufficiently small $\epsilon^{*}$, all terms involving $\epsilon^{*}$ in Eq. (32b) can be neglected. The boundary-value problem is then only implicitly dependent on $\epsilon^{*}$ ( $\rho$ and $\Lambda$ are functions of $\epsilon^{*}$ ). With these simplifications, Eqs. (32) can be written in the following matrix form for $\Lambda>0$ :

$$
\left[\begin{array}{cccc}
1 & 1 & 1 & 1 \\
p_{1} & \bar{p}_{1} & p_{2} & \bar{p}_{2} \\
b_{1} & \bar{b}_{1} & b_{2} & \bar{b}_{2} \\
g_{1} & \bar{g}_{1} & g_{2} & \bar{g}_{2}
\end{array}\right]\left[\begin{array}{l}
C_{1} \\
C_{3} \\
C_{2} \\
C_{4}
\end{array}\right]=0
$$


Expressions for the elements in the last two rows of the matrix, as determined from Eqs. (28) and (32), are written in the form

$$
b_{1}=b_{11}+i b_{12}, \quad b_{2}=b_{21}+i b_{22}, \quad g_{1}=g_{11}+i g_{12}, \quad g_{2}=g_{21}+i g_{22},
$$

where:

$$
\begin{gathered}
\begin{aligned}
b_{11}= & {\left[-\frac{1}{4}(1-\nu) \Lambda+2 \rho\right]\left[\rho \alpha_{1}-\left(1-\rho^{2}\right)^{1 / 2} \beta_{1}\right] } \\
& \quad+2^{-1 / 2}(1-\rho)^{1 / 2}(1+2 \rho)\left(\alpha_{1}^{2}-\beta_{1}^{2}\right)-(1-2 \rho) \alpha_{1} \beta_{1} 2^{1 / 2}(1+\rho)^{1 / 2}
\end{aligned} \\
\begin{aligned}
b_{12}=- & {\left[-\frac{1}{4}(1-\nu) \Lambda+2 \rho\right]\left[\left(1-\rho^{2}\right)^{1 / 2} \alpha_{1}+\rho \beta_{1}\right] } \\
& \quad-2^{-1 / 2}(1+\rho)^{1 / 2}(1-2 \rho)\left(\alpha_{1}^{2}-\beta_{1}^{2}\right)-(1+2 \rho) \alpha_{1} \beta_{1} 2^{1 / 2}(1-\rho)^{1 / 2} \\
b_{21}= & {\left[-\frac{1}{4}(1-\nu) \Lambda+2 \rho\right]\left[\rho \alpha_{2}-\left(1-\rho^{2}\right)^{1 / 2} \beta_{2}\right] } \\
& \quad-2^{-1 / 2}(1-\rho)^{1 / 2}(1+2 \rho)\left(\alpha_{2}^{2}-\beta_{2}^{2}\right)+(1-2 \rho) \alpha_{2} \beta_{2} 2^{1 / 2}(1+\rho)^{1 / 2} \\
b_{22}=- & \quad\left[-\frac{1}{4}(1-\nu) \Lambda+2 \rho\right]\left[\left(1-\rho^{2}\right)^{1 / 2} \alpha_{2}+\rho \beta_{2}\right] \\
& \quad+2^{-1 / 2}(1+\rho)^{1 / 2}(1-2 \rho)\left(\alpha_{2}^{2}-\beta_{2}^{2}\right)+(1+2 \rho) \alpha_{2} \beta_{2} 2^{1 / 2}(1-\rho)^{1 / 2}
\end{aligned} \\
g_{11}=-\frac{1}{4}(1-\nu) \Lambda \rho-(1+2 \rho) \alpha_{1} 2^{-1 / 2}(1-\rho)^{1 / 2}+(1-2 \rho) \beta_{1} 2^{-1 / 2}(1+\rho)^{1 / 2} \\
g_{12}=\frac{1}{4}(1-\nu) \Lambda\left(1-\rho^{2}\right)^{1 / 2}+(1-2 \rho) \alpha_{1} 2^{-1 / 2}(1+\rho)^{1 / 2}+(1+2 \rho) \beta_{1} 2^{-1 / 2}(1-\rho)^{1 / 2}, \\
g_{21}=-\frac{1}{4}(1-\nu) \Lambda \rho+(1+2 \rho) \alpha_{2} 2^{-1 / 2}(1-\rho)^{1 / 2}-(1-2 \rho) \beta_{2} 2^{-1 / 2}(1+\rho)^{1 / 2} \\
g_{22}=
\end{gathered}
$$

The matrix of Eqs. (36) is further transformed into a real-valued matrix by application of identities, the first of which is

$$
b_{1} C_{1}+\bar{b}_{1} C_{3} \equiv b_{11}\left(C_{1}+C_{3}\right)+i b_{12}\left(C_{1}-C_{3}\right) .
$$

The resulting set of equations is as follows:

$$
\left[\begin{array}{cccc}
1 & 0 & 1 & 0 \\
-\alpha_{1} & \beta_{1} & -\alpha_{2} & \beta_{2} \\
b_{11} & b_{12} & b_{21} & b_{22} \\
g_{11} & g_{12} & g_{21} & g_{22}
\end{array}\right]\left[\begin{array}{c}
C_{1}+C_{3} \\
i\left(C_{1}-C_{3}\right) \\
C_{2}+C_{4} \\
i\left(C_{2}-C_{4}\right)
\end{array}\right]=0
$$

The determinant of the matrix of Eqs. (39) is called $\Delta$. For a given value of $\nu, \Delta$ is a function of $\rho$ and $\Lambda$ only, and values of $\rho$ and $\Lambda$ for which $\Delta$ vanishes will determine the stability boundary for edge buckling. For arbitrary $\rho$ and $\Lambda, \Delta$ is algebraically complicated, and it is determined numerically without further algebraic simplification. A convenient numerical scheme is to fix a positive $\Lambda$ and to determine the zeros of $\Lambda$ as a function of $\rho$. Five such numerically-calculated zero points are shown as circled values in Fig. 3. It appears that $\Delta$ has two simple zeros for each positive $\Lambda$. It is assumed that only the lower branch of the curve in Fig. 3 is of physical significance as the stability boundary; the equilibrium of the shell for $\rho$-values above this lower line is assumed unstable. 


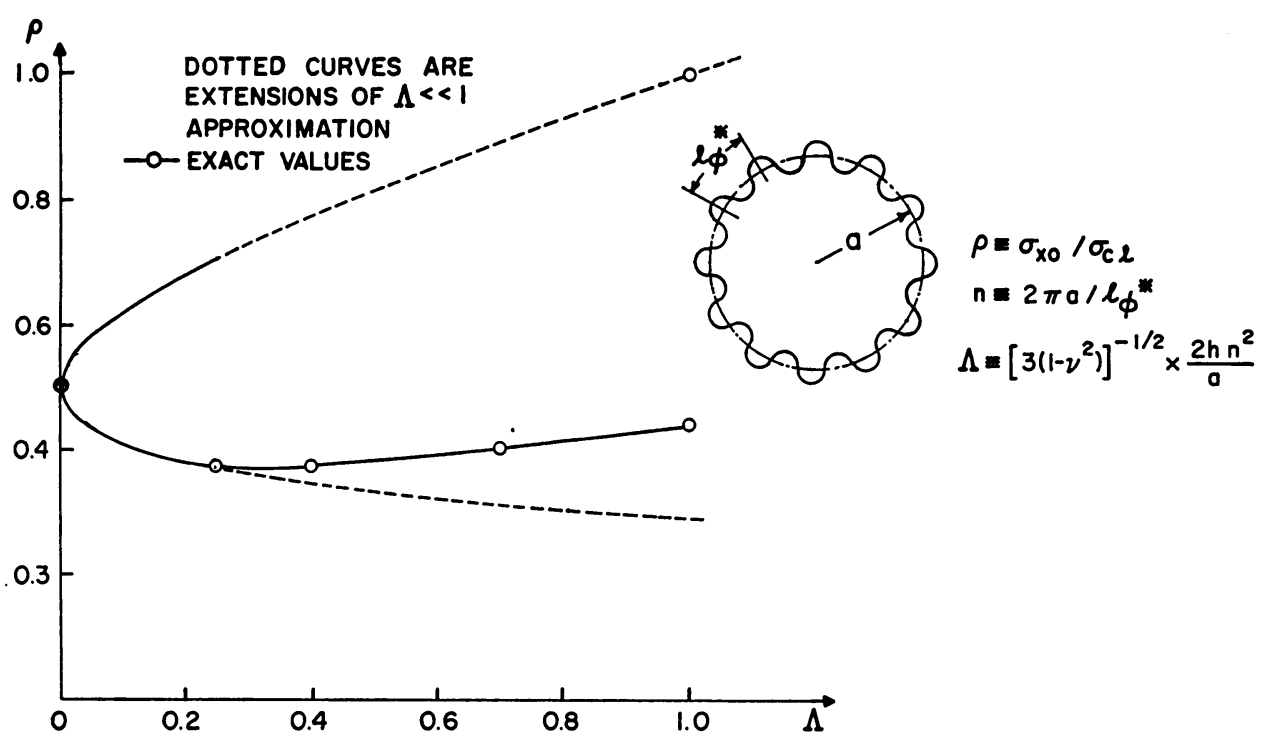

FIG. 3. Critical stress ratio $\rho$ vs. lobe number parameter $\Lambda$ for edge buckling of a semi-infinite cylindrical shell with a free edge under uniform axial compression $(\nu=0.3)$.

If $\Lambda$ is small, a simple, closed-form expression for $\Delta$ is found [8] by expanding the previously stated expressions for the elements of $\Delta$ into power series in $\Lambda$. After considerable algebraic manipulation, the following approximate expression, valid for $0<\Lambda^{2} \ll 1$, is obtained:

$$
\Delta=\left(\frac{1+\rho}{2}\right) \frac{\Lambda}{4}\left\{(1-2 \rho)^{2}-\frac{\Lambda}{4}\left[\left(-2+4 \nu+2 \nu^{2}\right)+\left(3-2 \nu-\nu^{2}\right) 2 \rho\right]+O\left(\Lambda^{2}\right)\right\}
$$

For $\Lambda>0$, the roots of $\Delta$ are given by Eq. (40) as the following curve:

$$
\Lambda=\frac{2(1-2 \rho)^{2}}{\left(-1+2 \nu+\nu^{2}\right)+\left(3-2 \nu-\nu^{2}\right) \rho} \text { for } \Lambda^{2} \ll 1
$$

This curve is shown in Fig. $\dot{3}$ for $\nu=0.3$ as solid for $\Lambda<0.25$ and as dotted for $\Lambda>0.25$. The remainder of the solid line passes through the circled values and is taken to be the correct lower branch of the curve for $\Lambda>0.25$. The minimum value of $\rho$ along this branch is computed from the portion of the curve shown to be $\rho=0.37$ at $\Lambda=0.32$.

The mode shape for edge buckling is now calculated. Since the amplitude of $w(x, \varphi)$ is indeterminate, and since the problem is invariant under a rigid-body rotation about the $x$-axis, we set $w(0,0)=1$. The mode shape $w(x, 0)$ for $x>0$ is found by taking the real part of Eq. (30). If the real numbers $w_{11}, \cdots, w_{22}$ are defined as

$$
\begin{aligned}
& w_{1} \equiv\left[-\rho+i\left(1-\rho^{2}\right)^{1 / 2}\right] e^{i \beta_{1} x} \equiv w_{11}+i w_{12} \\
& w_{2} \equiv\left[-\rho+i\left(1-\rho^{2}\right)^{1 / 2}\right] e^{i \beta_{2} x} \equiv w_{21}+i w_{22}
\end{aligned}
$$

and if a real-valued column vector $\left\{B_{K}\right\}$ is defined as

$$
\left\{B_{1}, B_{2}, B_{3}, B_{4}\right\}=\operatorname{Re}\left\{C_{1}+C_{3}, i\left(C_{1}-C_{3}\right), C_{2}+C_{4}, i\left(C_{2}-C_{4}\right)\right\},
$$


then it follows from Eqs. (30) and (38) that

$$
\begin{aligned}
w(x, 0)= & -\left\{\operatorname{Re}\left[C_{1} w_{1}+C_{3} \bar{w}_{1}\right] e^{-\alpha_{1} x}+\operatorname{Re}\left[C_{2} w_{2}+C_{4} \bar{w}_{2}\right] e^{-\alpha_{2} x}\right\} \\
= & -\left\{\left(B_{1} w_{11}+B_{2} w_{12}\right) e^{-\alpha_{1} x}+\left(B_{3} w_{21}+B_{4} w_{22}\right) e^{-\alpha_{2} x}\right\} \\
= & e^{-\alpha_{1} x}\left[B_{1}\left(\rho \cos \beta_{1} x+\left\{1-\rho^{2}\right\}^{1 / 2} \sin \beta_{1} x\right)\right. \\
& \left.+B_{2}\left(\rho \sin \beta_{1} x-\left\{1-\rho^{2}\right\}^{1 / 2} \cos \beta_{1} x\right)\right] \\
& +e^{-\alpha_{2} x}\left[B_{3}\left(\rho \cos \beta_{2} x+\left\{1-\rho^{2}\right\}^{1 / 2} \sin \beta_{2} x\right)\right. \\
& \left.+B_{4}\left(\rho \sin \beta_{2} x-\left\{1-\rho^{2}\right\}^{1 / 2} \cos \beta_{2} x\right)\right] .
\end{aligned}
$$

When $\rho$ and $\Lambda$ are on the stability boundary, then there will exist column vectors $\left\{B_{K}\right\}$ which satisfy Eqs. (39). Using the normalization condition derived from Eq. (44),

$$
w(0,0)=-\left(B_{2}+B_{4}\right)\left\{1-\rho^{2}\right\}^{1 / 2}=1
$$

and the first of Eqs (39),

$$
B_{1}+B_{3}=0 \text {, }
$$

we derive the following expressions $B_{1}$ and $B_{2}$ from the second and fourth of the Eqs. (39):

$$
\begin{aligned}
& B_{1}=\left(1-\rho^{2}\right)^{-1 / 2} \frac{\beta_{2}\left(g_{12}-g_{22}\right)-g_{22}\left(\beta_{1}-\beta_{2}\right)}{\left(\alpha_{2}-\alpha_{1}\right)\left(g_{12}-g_{22}\right)-\left(\beta_{1}-\beta_{2}\right)\left(g_{11}-g_{21}\right)}, \\
& B_{2}=\left(1-\rho^{2}\right)^{-1 / 2} \frac{g_{22}\left(\alpha_{2}-\alpha_{1}\right)-\beta_{2}\left(g_{11}-g_{21}\right)}{\left(\alpha_{2}-\alpha_{1}\right)\left(g_{12}-g_{22}\right)-\left(\beta_{1}-\beta_{2}\right)\left(g_{11}-g_{21}\right)} .
\end{aligned}
$$

Edge buckling for $\nu=0.3$ occurs at $\rho=0.37$ and $\Lambda=0.32$; functional values are calculated from Eqs. (28) and Eqs. (45), (46) and (47) to be

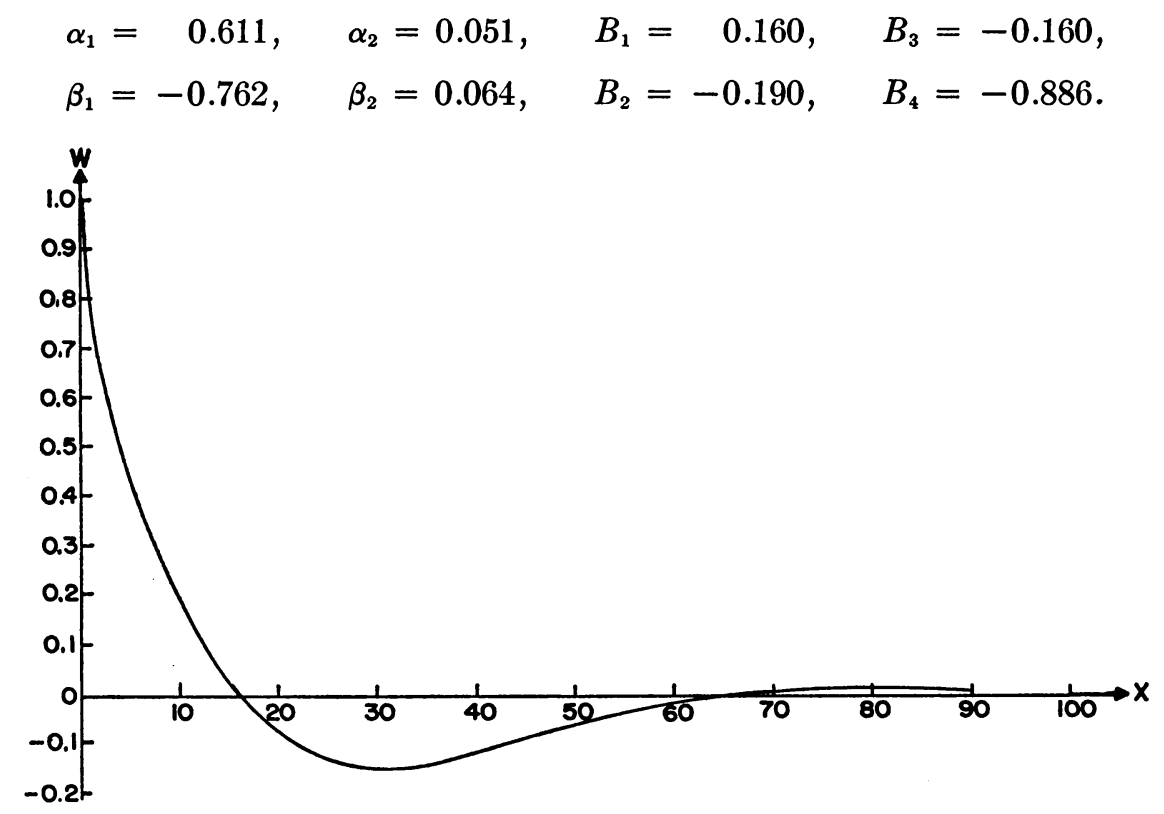

Fig. 4. Lateral displacement $w$ in free edge buckling as a function of axial coordinate $x(\nu=0.3)$. 
Equation (44) takes the following form for these values:

$$
\begin{aligned}
& w(x, 0)=e^{-0.611 x}[0.234 \cos (0.762 x)-0.079 \sin (0.762 x)] \\
& +e^{-0.051 x}[0.766 \cos (0.064 x)-0.477 \sin (0.064 x)] .
\end{aligned}
$$

Equation (48) is plotted in Fig. 4.

Acknowledgment. The authors are grateful to Mrs. Wen-Yi Shih Pi who checked the algebra and performed the computations for the figures.

\section{REFERENCES}

1. Y. C. Fung and E. E. Sechler, Instability of thin elastic shells, Structural Mechanics, Proceedings of the First Symposium on Naval Structural Mechanics, ed. by J. N. Goodier and N. J. Hoff, Pergamon Press, N. Y., 1960, pp. 115-168

2. N. J. Hoff, Buckling of thin shells, Von Kármán 80th Anniversary Lecture, May 11, 1961, to be published by the Institute of the Aerospace Sciences; see also SUDAER No. 114, Department of Aeronautical Engineering, Stanford University, 1961

3. L. H. Donnell, A new theory for the buckling of thin cylinders under axial compression and bending, Trans. A. S. M. E., 56, (1934) 795-806

4. W. Nachbar, Discontinuity stresses in pressurized thin shells of revolution Report LMSD-48483, Lockheed Missiles and Space Division, Sunnyvale, California, 1959

5. S. Timoshenko and S. Woinowsky-Krieger, Theory of plates and shells, second edition, McGrawHill, New York, 1959, pp. 432, 508, 512, 518, 523

6. A. E. H. Love, $A$ treatise on the mathematical theory of elasticity, fourth edition, Dover, New York, 1944, pp. 517-522

7. W. Nachbar, Characteristic roots for Donnell's equations with uniform axial prestress, SUDAER No. 108, Department of Aeronautical Engineering, Stanford University, July 1961, to be published in the Journal of Applied Mechanics

8. W. Nachbar and N. J. Hoff, On edge buckling of axially-compressed, circular cylindrical shells, SUDAER No. 115, Department of Aeronautical Engineering, Stanford University, November 1961 